\title{
Intersubband Transitions in InAs/AlSb Quantum Wells
}

J. Li, ${ }^{1)}$ K. Kolokolov, ${ }^{1)}$, C. Z. Ning, ${ }^{1,)}$ D. C. Larraber, ${ }^{2)}$ G. A. Khodaparast, ${ }^{2)}$ J. Kono, ${ }^{2)}$ K. Ueda, ${ }^{3)}$ Y. Nakajima, ${ }^{3)}$ S. Sasa, ${ }^{3)}$ and M. Inoue ${ }^{3)}$

${ }^{1)}$ Center for Nanotechnology, NASA Ames Research Center, Moffett Field, CA 94035

${ }^{2)}$ Department of Electrical and Computer Engineering, Rice University, Houston, TX 77005

${ }^{3)}$ Department of Electrical Engineering, Osaka Institute of Technology, Osaka, Japan

\begin{abstract}
We have studied intersubband transitions in InAs/AlSb quantum wells experimentally and theoretically. Experimentally, we performed polarization-resolved infrared absorption spectroscopy to measure intersubband absorption peak frequencies and linewidths as functions of temperature (from $4 \mathrm{~K}$ to room temperature) and quantum well width (from a few $\mathrm{nm}$ to $10 \mathrm{~nm}$ ). To understand experimental results, we performed a self-consistent 8 -band $\mathbf{k} \cdot \mathbf{p}$ band-structure calculation including spatial charge separation. Based on the calculated band structure, we developed a set of density matrix equations to compute TE and TM optical transitions self-consistently, including both interband and intersubband channels. This density matrix formalism is also ideal for the inclusion of various many-body effects, which are known to be important for intersubband transitions. Detailed comparison between experimental data and theoretical simulations is presented.
\end{abstract}

\section{INTRODUCTION}

Antimonide-based quantum wells formed by three nearly lattice matched binaries, InAs, $\mathrm{GaSb}, \mathrm{AlSb}$, and their alloys have attracted much interest due to their wide range of

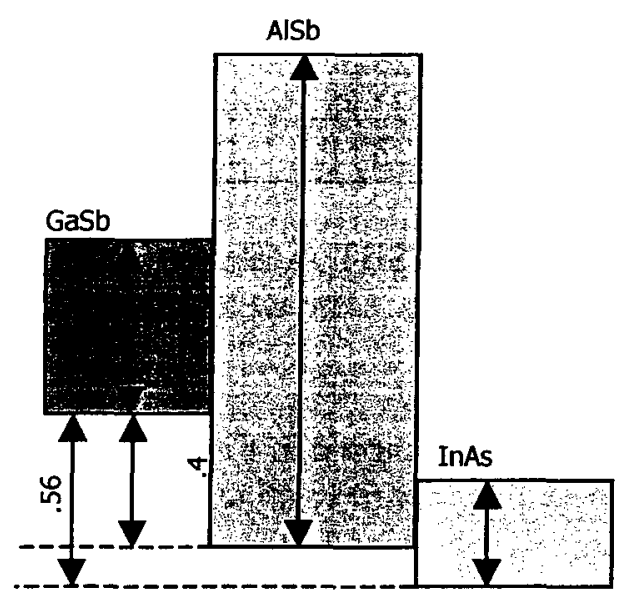
potential applications in devices such as long wavelength detectors $[1,2]$ and lasers [3]. Of special interest for optical applications are the extremely large conduction band offsets in InAs/AlSb heterostructures. Due to the large band gap difference and the special band-edge lineups (see Fig. 1), the conduction band well defined by the $\Gamma$-points of the two materials is as deep as $2 \mathrm{eV}$. Obviously, such a deep well offers great flexibility in realizing intersubband transitions (ISBTs) of a wide wavelength range, i.e., from the near-infrared (NIR) all the way to the far-infrared (FIR), or terahertz (THz) region.

It was recently shown theoretically that a proper multi-well design using deep InGaAsAlAsSb-InP quantum wells allows completely intersubband-based, optically-pumped $\mathrm{THz}$ 
generation $[4,5]$. The advantage of such a scheme is that the pumping laser can be another compact semiconductor laser operating at $1.5 \mu \mathrm{m}$, instead of a $\mathrm{CO}_{2}$ laser commonly used for GaAs-based quantum wells. Similarly, InAs/A1Sb quantum wells can be used for intersubband-pumped long-wave generation.

As a first step towards realizing such a $\mathrm{THz}$ source, we have conducted a systematic study of optical transitions in InAs/AlSb quantum wells for a wide range of well widths. Specifically, we studied intersubband absorption spectra with varying temperatures and well widths. A large red shift of the ISBT frequency with increasing temperature was observed, which was accompanied by an increase of the ISBT linewidth. To explain these experimental results, we developed a comprehensive theory that includes many-body Coulomb interactions, band structure effects based on an 8-band $\mathbf{k} \cdot \mathbf{p}$ theory, and line broadening mechanisms due to phonon scattering and interface roughness scattering. As we will demonstrate in the following, this theory explains the experimental results very well.

\section{EXPERIMENTAL METHODS AND RESULTS}

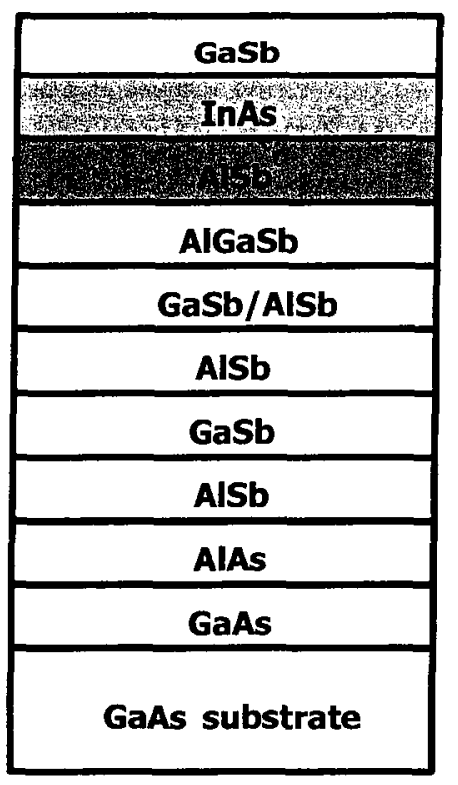

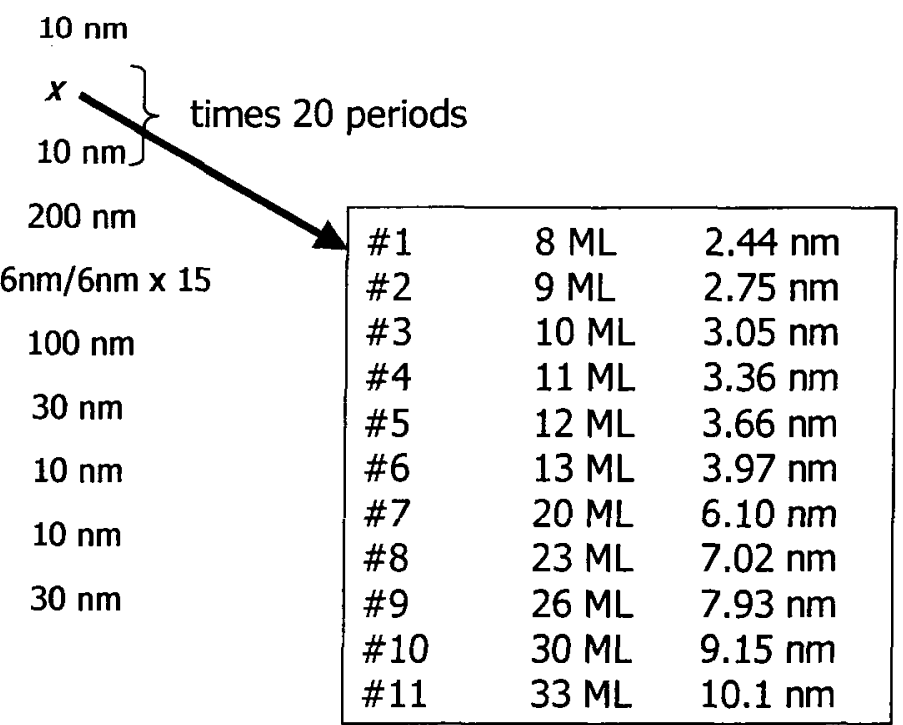

FIGURE 2. Typical sample structure used for experimental ISBT measurements. The active region consists of 20 periods of InAs/AlSb quantum wells. The well widths for the 11 samples investigated are indicated in the square box.

The typical sample structure used in the experiments is shown in Fig. 2. We grew and measured InAs/AISb multiple quantum wells with various well widths as indicated in Fig. 2. The samples were grown by MBE (at the Osaka Institute of Technology) and experimentally characterized by Fourier-transform infrared (FTIR) spectroscopy (at Rice University). A schematic diagram of the experimental geometry used for multi-bounce, edge-coupled absorption measurements is shown in Fig. 3. The incident IR beam was linearly polarized and we recorded the $\mathrm{p} / \mathrm{s}$ ratio of the transmitted beam (only the $\mathrm{p}$ component is intersubband-active). 


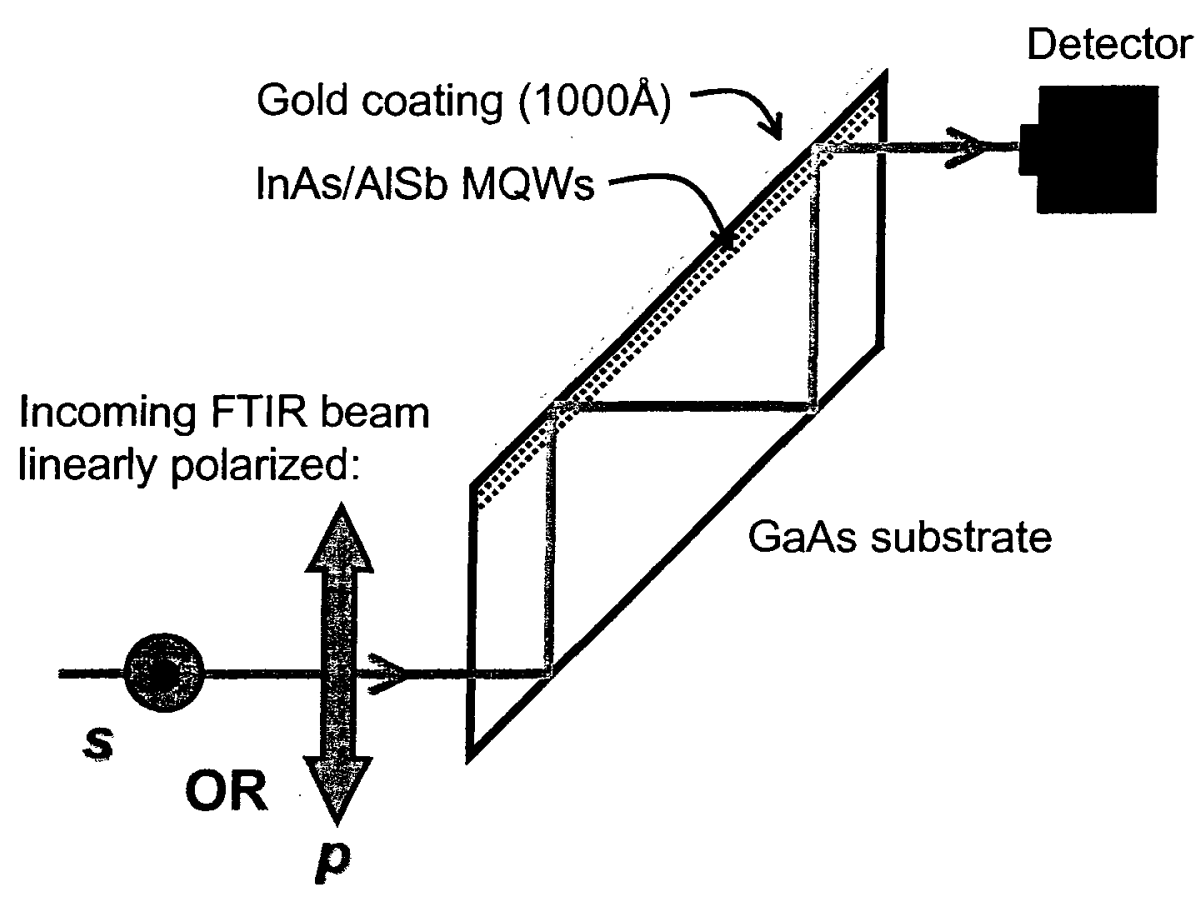

FIGURE 3. Schematic diagram of the experimental setup for intersubband absorption measurements.

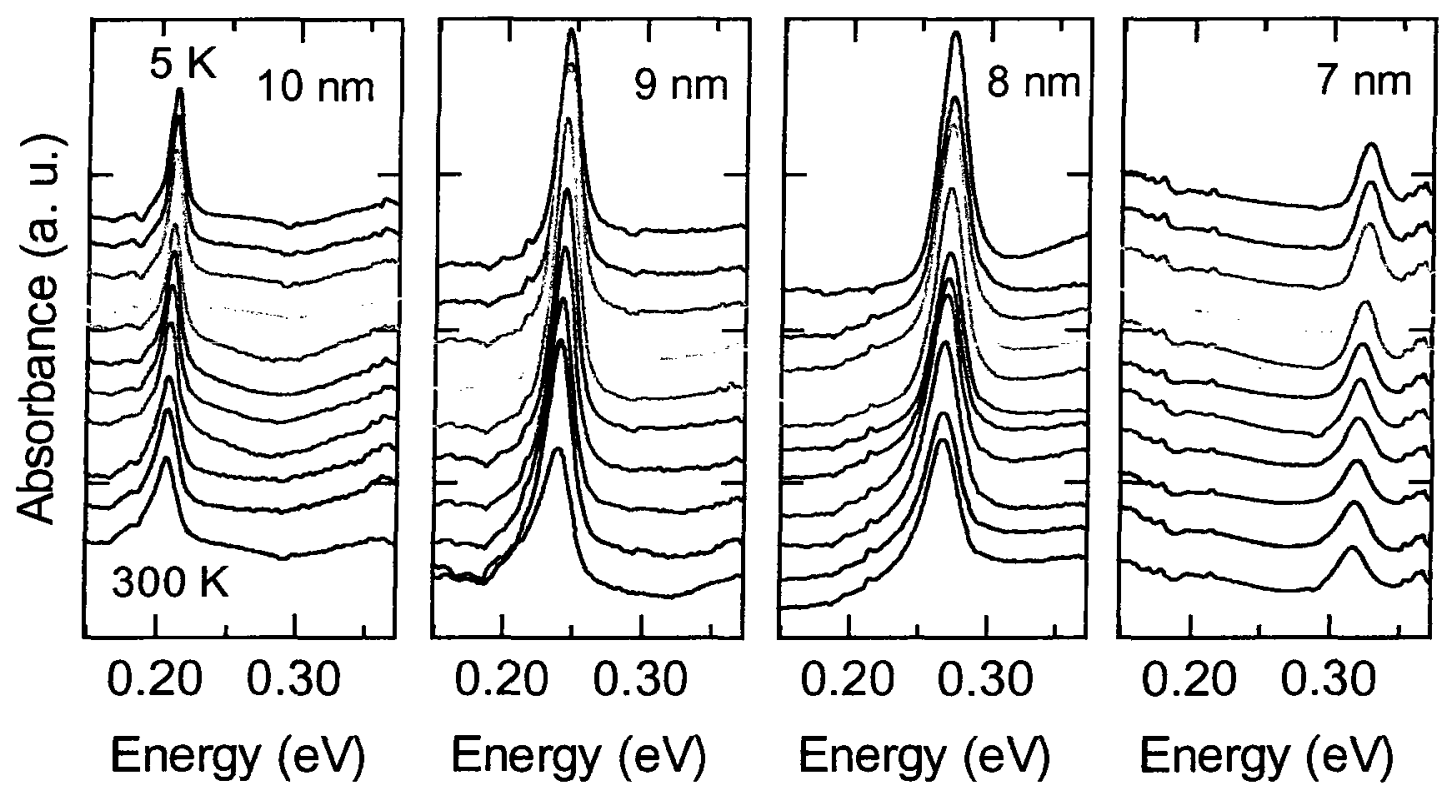

FIGURE 4. Experimentally measured ISBT spectra for the 4 widest quantum wells. For each well width, absorption was measured at 11 temperatures from $5 \mathrm{~K}$ to $300 \mathrm{~K}$.

FTIR absorption spectra for the four widest wells are shown in Fig. 4. They exhibit resonances due to ISBTs within the conduction band, i.e., $E_{1} \rightarrow E_{2}$. There are a few features that are worth noting. First, for a given well width, the ISBT peak shows a red shift as the temperature is raised. The amount of the red shift depends on the well 
width and varies for the four samples between $7 \mathrm{meV}$ and $11 \mathrm{meV}$ in the temperature range of $5 \mathrm{~K}$ to $300 \mathrm{~K}$. The temperature dependence of the ISBT peak positions is summarized in Fig. 5. The second feature to note in Fig. 4 is the non-monotonic change of the absorption strength with well width. The absorption peaks for the 8 and $9 \mathrm{~nm}$ samples are larger than that for the 7 and $10 \mathrm{~nm}$ samples while the $7 \mathrm{~nm}$ sample shows the weakest absorption. For wells narrower than $7 \mathrm{~nm}$, we were unable to observe ISBTs. The significant reduction of absorption at $7 \mathrm{~nm}$ seems to be a precursor of the disappearance of ISBT in narrower wells. There are a few possible reasons for the disappearance, with the most likely reason being the decrease of electron density in the InAs layer with well width reduction. Note that the samples investigated are not intentionally doped. It is known that a high density of electrons always exists in notintentionally-doped InAs/AlSb heterostructures, whose sources are somewhat controversial. One of the dominating electron sources is deep impurity states in the AlSb barriers [6-8]. These impurities lie higher than the ground state of the InAs well for wide wells, making it possible for electrons to transfer from the AlSb layers to the InAs layer. When the well width become narrower, the ground state energy increases and such electron transfer becomes less efficient. It is therefore possible that the ground state energy eventually becomes higher than the impurity level in the AlSb layer, inhibiting any electron transfer from AlSb layers. Other reasons also lead to the decrease of intersubband absorption, such as decrease of the dipole moments and increase of interface roughness scattering with decreasing well width.

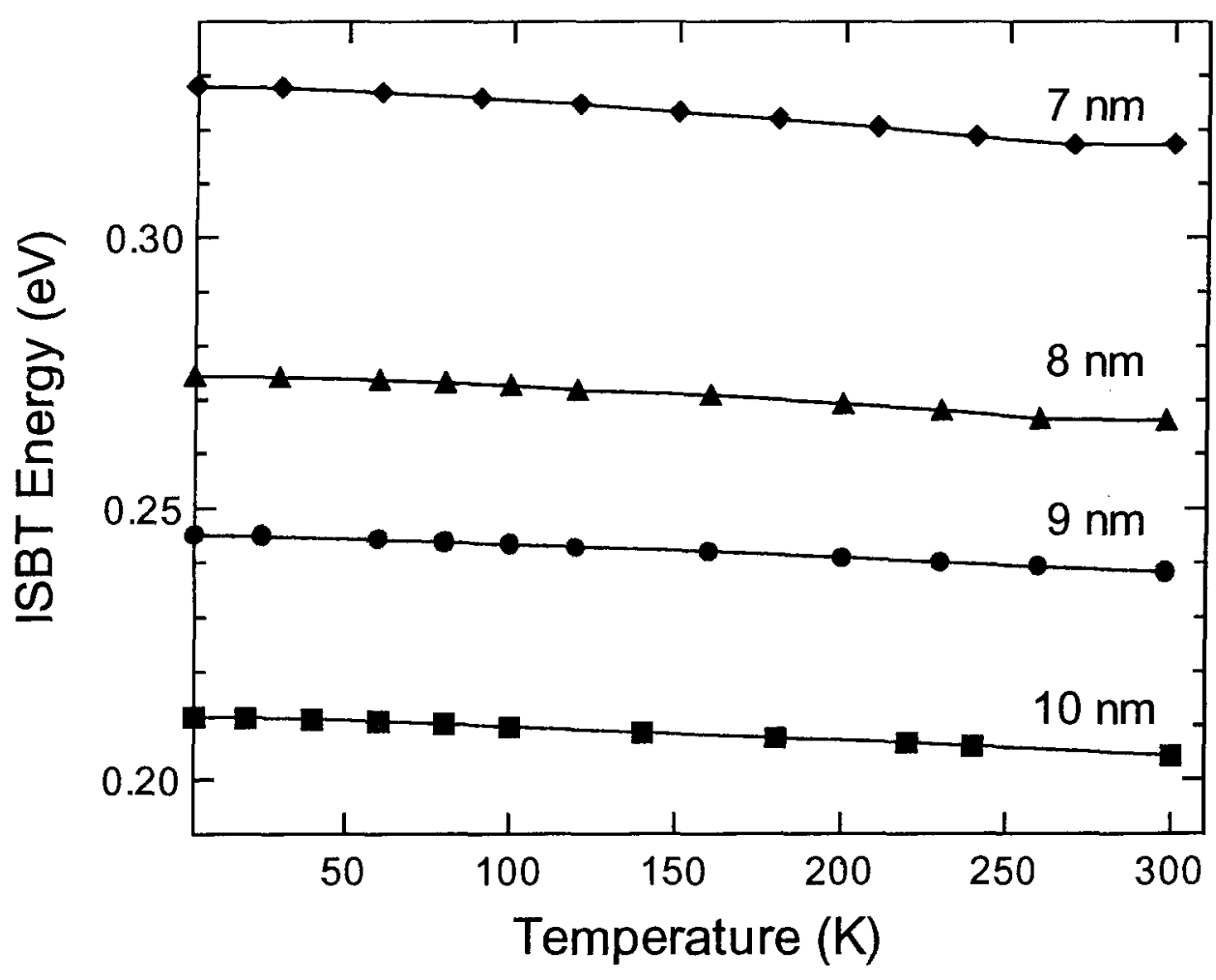

FIGURE 5. ISBT absorption peak energy vs. temperature extracted from the data in Fig. 4 by Lorentzian fitting near the absorption peak. 


\section{THEORY}

To design antimonide-based quantum well structures for long wavelength generation and to understand the experimental results summarized above, we developed a comprehensive microscopic theory for InAs/AlSb quantum wells and superlattice structures. In the past, intersubband and other experimental results in this materials system have been dealt with using an effective one-band model with a non-parabolicity parameter [9], or an 8-band model that was also used to derive various transition matrix elements [10]. Another important aspect of intersubband transitions is concerned with many-body effects. While it has long been recognized that various many-body effects, such as depolarization and excitonic shifts, are important for ISBTs [11,12], systematic treatment and study of those effects have become available only recently $[13,14]$. The early theory by Ando [11] led to simple resonance frequency shifts due to depolarization and excitonic effects, denoted by $\alpha$ and $\beta$, respectively. While this formula is simple and powerful, it is applicable to parabolic bands only. Furthermore, it cannot explain the cancellation of the entire exchange contribution when applied to an exactly twodimensional system with a parabolic band. This is also true for other later generalizations of Ando's theory to non-parabolic cases. A more systematic approach based on Green's function was taken by Huang et al. [14], where all major many-body effects were treated on more equal footings. This treatment was done for an effective one bulk-band model. In our treatment, we base our approach on the density matrix formalism that has been well established over the past decade for interband semiconductor optics $[15,16]$. The theory, known as semiconductor Bloch equations, allows a systematic approximation of scattering terms, which are important for describing dephasing and linewidth. Our theoretical treatment combines band structure calculations with such a microscopic many-body theory.

For band structure calculations, we adopt the standard 8-band $\mathbf{k} \cdot \mathbf{p}$ theory with the Hartree self-consistent potential. The electron wave function can in general be written as

$$
\Psi(\mathbf{r}, z)=\frac{1}{\sqrt{A}} \sum_{n, \mathbf{k}_{\|}} e^{i \mathbf{r} \bullet \mathbf{k}_{\|}} a_{n, k} \mathbf{u}(r) \bullet \mathbf{F}_{\mathbf{k}_{\|}}^{n}(z)
$$

where the Bloch functions for the 8 band at the $\Gamma$-point and the corresponding envelope functions are given respectively by

$$
\begin{aligned}
& \mathbf{u}=\left(u_{1 / 2}^{c}, u_{-1 / 2}^{c}, u_{3 / 2}^{v}, u_{1 / 2}^{v}, u_{-1 / 2}^{v}, u_{-3 / 2}^{v}, u_{1 / 2}^{s}, u_{-1 / 2}^{s}\right) \equiv\left(u_{1}, \ldots, u_{8}\right), \\
& \mathbf{F}_{\mathbf{k}_{\|}}^{n}=\left(F_{1, \mathbf{k}_{\mid l}}^{n}, \ldots, F_{8, \mathbf{k}_{\| 1}}^{n}\right) .
\end{aligned}
$$

The envelope functions are solved from the 8-band Schroedinger equation

$$
\left(H_{8 \times 8}+V_{H S}+V_{H}\right) F_{\mathbf{k}_{\|}}^{n}=E_{n}^{(0)}\left(\mathbf{k}_{\|}\right) F_{\mathbf{k}_{\|}}^{n}
$$

with $V_{H S}$ being the heterostructure potential and $V_{H}$ the self-consistent Hartree potential which includes both doping density profile and the profile of free mobile charges. This 
8-band matrix Schroedinger equation determines the energies and wave functions of all the confined states.

Next, the many-body Hamiltonian is expressed in terms of annihilation and creation operators $a_{n, k}$ and $a_{n, k}^{+}$. The remaining derivation of the equation of motion is straightforward and same as in $[15,16]$. A set of equations is finally derived for the population variables $f_{n}(k)=\left\langle a_{n, k}^{+} a_{n, k}\right\rangle$ and for the polarization variables $p_{l m}(k)=\left\langle a_{l, k}^{+} a_{m, k}\right\rangle$. Details of derivation including various scattering terms will be given elsewhere [17]. This set of equation is analogous to the semiconductor Bloch equations for the interband case $[15,16]$. For linear optical problems, such as optical absorption, the equations for the off-diagonal density matrix $p_{l m}(k)$ can be solved in the Fourier domain using matrix inversion. The total medium polarization is then written as

$$
P(\omega)=\frac{1}{A} \sum_{j l, \mathbf{k}} d_{j l}(\mathbf{k}) p_{j l}(\mathbf{k}, \omega)=\varepsilon_{0} \varepsilon_{b} \chi(\omega) E(\omega)
$$

where $E(\omega), \varepsilon_{0}$, and $\varepsilon_{b}$ are the electric field and vacuum and background semiconductor dielectric constants, respectively. The $\chi(\omega)$ is the linear susceptibility, which is given by

$$
\chi(\omega)=\frac{1}{2 \pi^{2} W} \sum_{j l} Q^{D}{ }_{j l}(\omega) \int \frac{\left|d_{j l}\left(\mathbf{k}_{\|}\right)\right|^{2}\left[f_{j}\left(\mathbf{k}_{\|}\right)-f_{l}\left(\mathbf{k}_{\|\|}\right)\right]}{-i \Gamma_{j, l}\left(\mathbf{k}_{\|}\right)+\left[E_{j \mathbf{k}_{\|}}^{0}+\Delta_{j j \mathbf{k}_{\|}}-\left(E_{i \mathbf{k}_{\|}}^{0}+\Delta_{l / \mathbf{k}_{\|}}\right)-\hbar \omega\right]} Q_{j l}^{V}\left(\mathbf{k}_{\|}\right) d^{2} \mathbf{k}_{\|}
$$

This expression contains all three major many-body effects that are important for the intersubband transitions. They are the so-called depolarization effect represented by $Q^{D}{ }_{j l}(\omega)$, the exchange interaction induced self-energy: $\Delta_{j j k}$, which modifies the band structure $\left(E^{0}{ }_{j k}\right)$, and the vertex or "excitonic" effect, $Q^{V}{ }_{j l}$. The linewidth $\Gamma_{j, l}$ in our current treatment includes phonon scattering and interface roughness scattering.

\section{MANY-BODY EFFECTS IN INTERSUBBAND TRANSITIONS}

As we mentioned in the last section, there are three major many-body effects known in intersubband transitions in quantum wells. The exchange interaction leads to two opposite shifts. Both the self-energy correction and vertex terms are due to the exchange interaction. While the former leads to a blue shift, the latter leads to a redshift. The results of the interplay among these many-body effects in general are complicated, especially for materials with strongly non-parabolic bands, such as InAs.

Such a complicated interplay is demonstrated in Fig. 6, where intersubband absorption spectra for a $10 \mathrm{~nm}$ InAs/AlSb quantum well are plotted. Here, to compare and identify individual effects, we artificially turned on and off some effects and the resulting absorption spectra are shown in five separate panels, Figs. 6(a)-6(e). Figure 
6(a) shows the single-particle intersubband absorption spectrum without including any many-body effects. As expected, the spectrum is broad and asymmetric and has a flat top. What is interesting here is that the spectrum is broadened to the high frequency side, instead of the low frequency side as expected from non-parabolicity. We attribute this behavior to phonon scattering, the rate of which is $\mathbf{k}$-dependent.

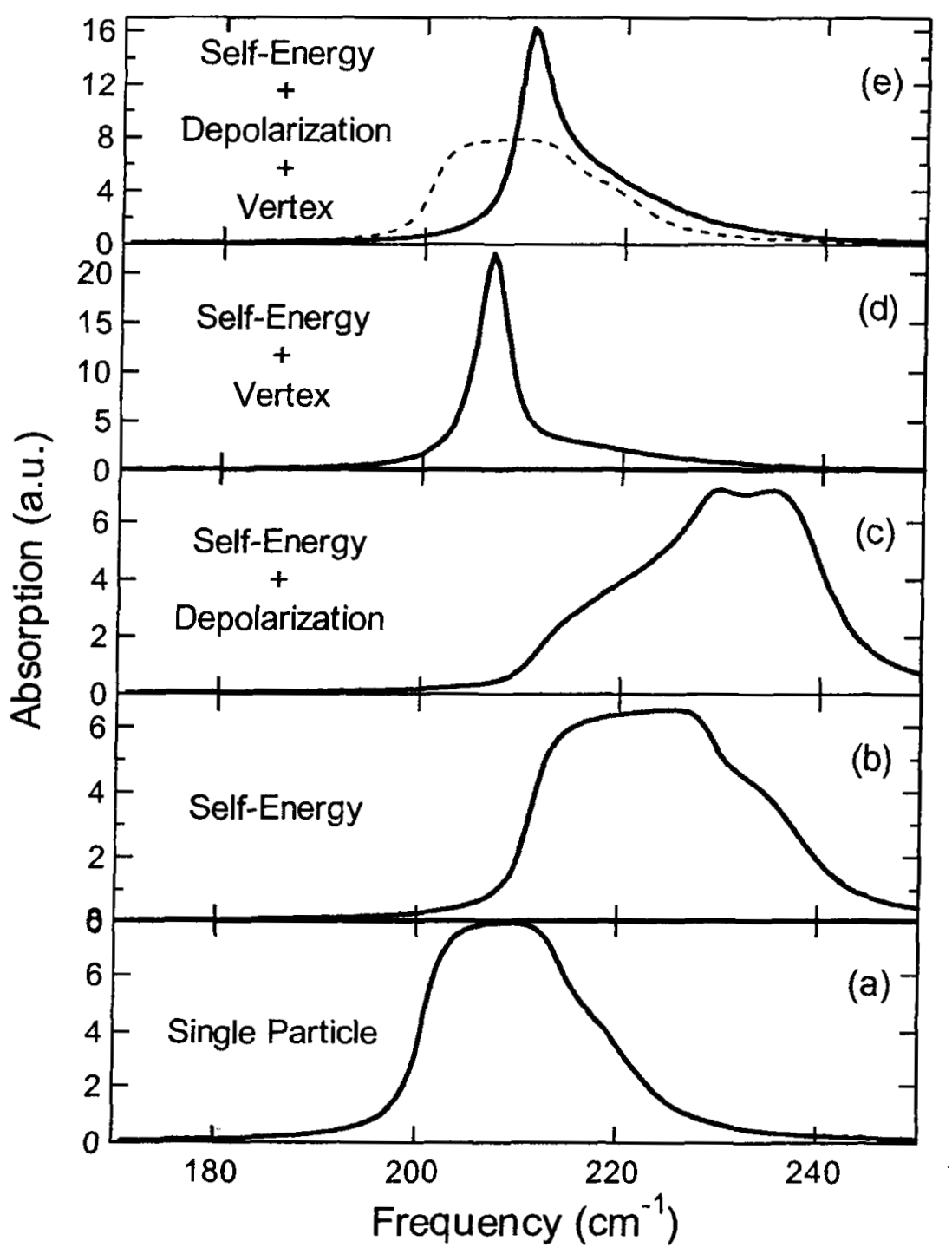

FIGURE 6. Calculated ISBT absorption spectra for InAs/AlSb quantum well (well width $=10 \mathrm{~nm}$ ) for density $8 \times 10^{11} \mathrm{~cm}^{-2}$ showing comparison of various many-body effects at $T$ $=4.5 \mathrm{~K}$. The dashed line in (e) is the single particle result, the same as the trace in (a).

Adding the self-energy correction due to the exchange interaction leads to a blue shift, as shown in Fig. 6(b). We see that the spectral lineshape is almost unchanged from (a), except that it is more broadened and blue shifted. The spectrum becomes broader due to k-dependent self-energy. The quite large blue shift is due to a downward shift of the lowest subband, which is populated, while there is almost no shift of the first excited state (second subband), which is not populated. 
As is well known and shown in Fig. 6(c), adding the depolarization effect leads to a further blue shift. However, in addition, the line becomes even broader. This is different from the common belief that the depolarization effect collapses the spectrum into a very narrow peak [10]. Even though we do see this collapse in some other cases, the situation in general is more complicated. We will leave a more complete discussion of this phenomenon to a future publication [17].

Next, we add only the vertex and self-energy corrections to the free-carrier model and the result is shown in Fig. 6(d) (the depolarization effect is not included here). We now see a significant narrowing of the absorption line. At the same time, the peak is shifted to the red side. By comparing Figs. 6(c) and 6(d), we can clearly see the separate roles played by the depolarization effect and vertex correction. It is the vertex correction, rather than the depolarization effect, that collapses the very broad spectrum into a narrow, exciton-like peak. At the same time, the absorption strength increases quite significantly. The position of this peak is between the low energy edge of the single-particle spectrum (a) and that of the self-energy-corrected spectrum (b), not at the low energy edge of the self-energy-corrected spectrum as one might expect as a signature of the Fermi edge singularity.

Finally, the full many-body spectrum is obtained [Fig. 6(e)] by including the depolarization effect to the result of Fig. 6(d). We see that the full many-body spectrum is more similar to that in (d) than that in row (c) both in peak position and spectral shape. This also suggests that many-body effects in this situation are dominated by the vertex term. Another important fact is that there are some cancellations; the final spectrum does not show the huge blue shift shown in (c). To compare the single-particle spectrum with that of full many-body, we copied the single-particle spectrum of (a) to (e) (the dashed line). We clearly see that the overall many-body effects in this case are to increase the absorption peak and move the spectrum slightly to the blue side.

\section{THEORY-EXPERIMENT COMPARISON}

As a first step towards understanding experimental ISBT absorption spectra, we computed the band structure of confined states in InAs/AlSb quantum wells at different temperatures. The difference in band-edge energies, $E_{12}$, of the first two subbands is plotted in Fig. 7 as a black curve. We see that it increases with increasing temperature, opposite to what was observed experimentally (Fig. 5). Next we computed simple absorption spectra by assuming a Lorentzian lineshape with a width of $6 \mathrm{meV}$ (red curve) or $12 \mathrm{meV}$ (blue curve) and plotted the peak position versus temperature. In both cases, we still see a blue shift, contrary to the red shift seen in the experiment. The main reason for the blue shift is the decrease of the effective mass with increasing temperature. The standard Kane theory relates the band edge effective mass with the band gap. As the temperature is raised, the band gap decreases according to the Varshni formula, leading to a significant decrease in effective mass. Such a significant reduction in effective mass leads to an increase in energy difference of the first two states. However, such a mass reduction seems to be inconsistent with experimental measurements. Experimentally, it is well known that measured temperature-dependent mass changes are very small, totally inconsistent with what is predicted from temperature dependent band gaps [18]. 


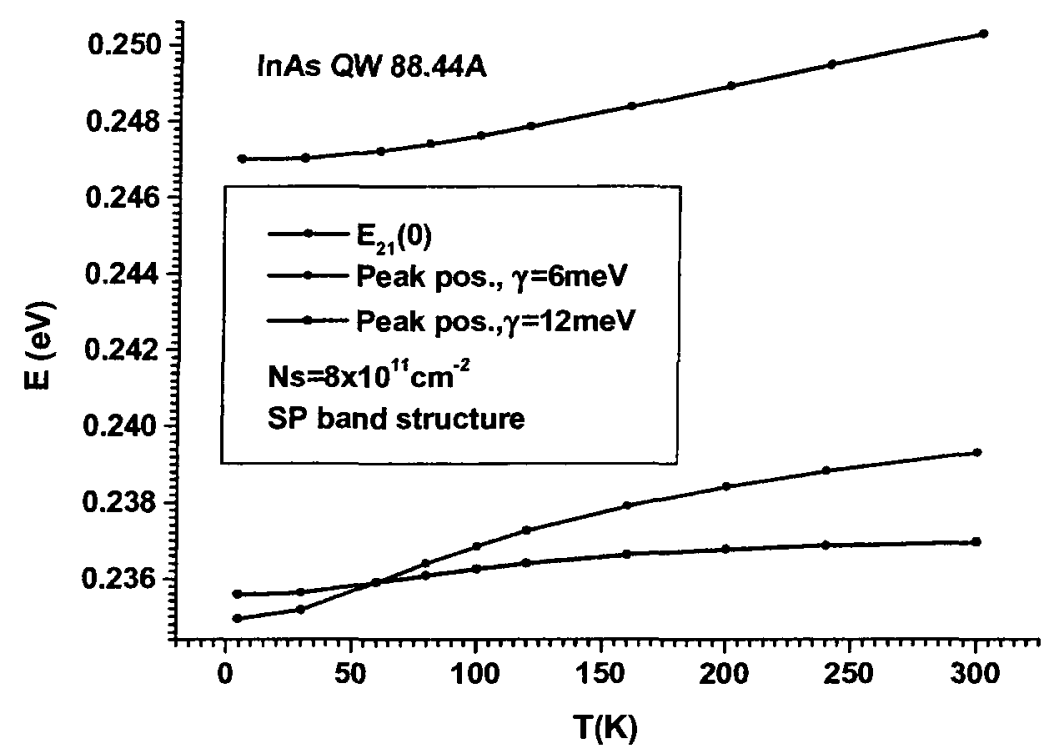

FIGURE 7. Band-edge difference of the ground and first excited states (black curve) and intersubband absorption peak positions (red and blue curves) versus temperature.

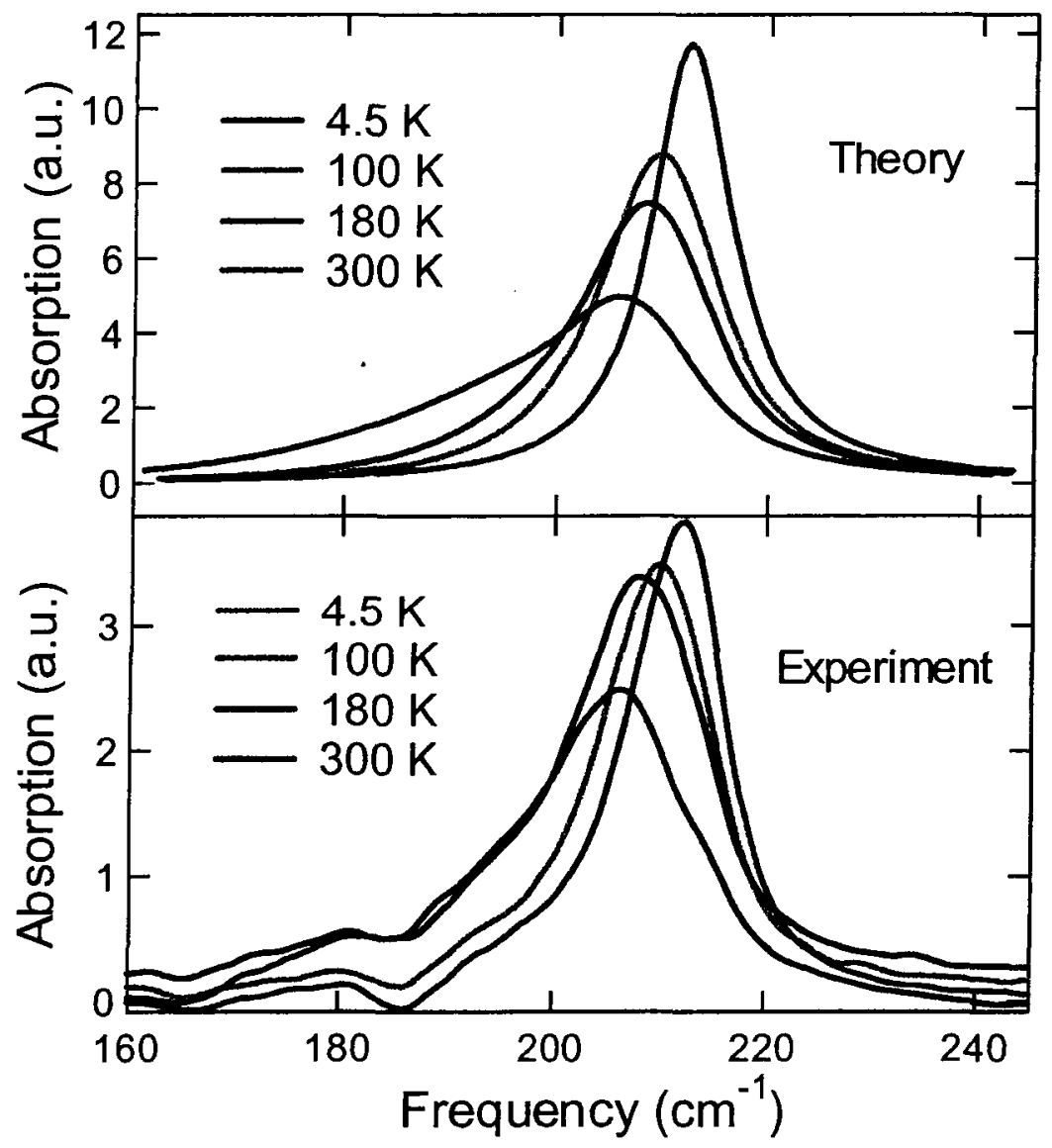

FIGURE 8. Intersubband absorption spectra for a $10 \mathrm{~nm}$ InAs/AlSb quantum well at four different temperatures as calculated theoretically (upper panel) and measured experimentally (lower panel). The four curves in the order of decreasing peak values correspond to $4.5,100,180$, and $300 \mathrm{~K}$, respectively. 
It turns out that the red shift of ISBT peak position with increasing temperature is a quite delicate issue. Similar red shifts have been observed in GaAs/AlGaAs systems [19] and studied quite extensively by Manasreh et al. [20]. The red shifts observed in most experiments [19-20] for GaAs are much smaller (about 3-4 meV) than in our experiments for InAs (with one exception [21]). This difference is related to the much smaller non-parabolicity in GaAs, but a full discussion of such a red shift needs to include many-body effects [14]. For InAs/A1Sb quantum wells, there is an added complication due to temperature-dependent carrier densities [6-8]. Unfortunately, the issue of density change with temperature is still an unsettled issue. In the following, we will fix the density when varying temperature. For the band edge mass change with temperature, we used experimentally measured data [18] instead of the Kane theory. In Fig. 8, calculated spectra at four different temperatures are shown (upper panel) together with the experimentally measured spectra (lower panel). The calculated spectral features agree with the experimental data quite well, including the peak position shifts with temperature and the linewidth change. However, the change of peak values of the absorption with temperature shows some deviation from experiments. For example, the peak values measured experimentally for $100 \mathrm{~K}$ and $180 \mathrm{~K}$ are almost the same, while the theoretical values are quite different. We believe that this is due to the inappropriate treatment of carrier density change with temperature.

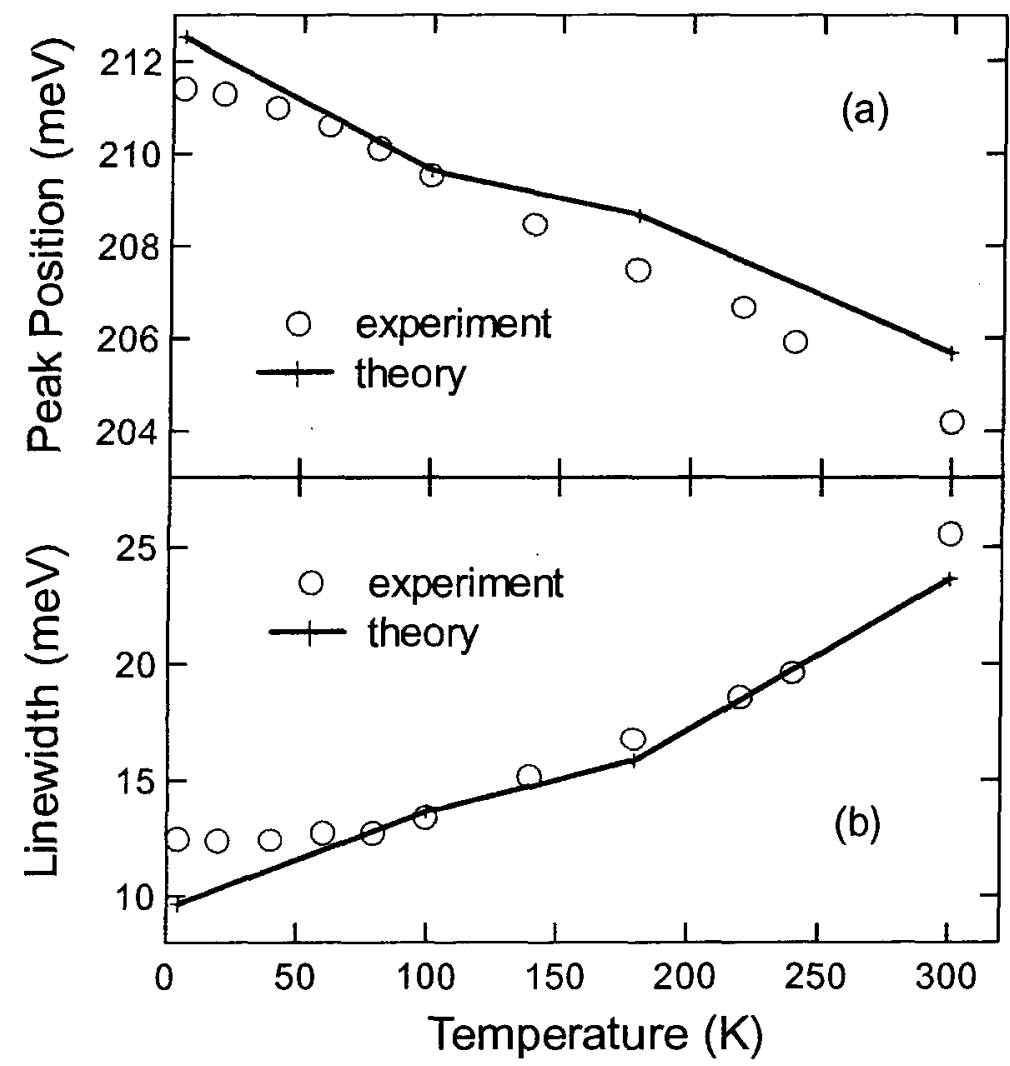

FIGURE 9. Temperature dependence of linewidth and peak frequency. Experimental values are denoted by circles while crosses with solid lines drawn through are theoretical calculation. Electron density is $8 \times 10^{10} \mathrm{~cm}^{-2}$. 
In Fig. 9, we compare theory and experiment on the temperature dependences of linewidth and peak frequency for the $10 \mathrm{~nm}$ InAs/AISb quantum well. In the theoretical calculation, we also included the LO phonon and interface roughness scatterings in addition to a $0.2 \mathrm{meV}$ homogeneous linewidth. We use Ando's model to take into account the interface scatterings [22]. This model uses two phenomenological constants to describe the magnitude of the interface roughness $(\Delta)$ and correlation length $(\Lambda)$ of interface fluctuation. In our modeling, we use the values of $0.4 \mathrm{~nm}$ and $6.1 \mathrm{~nm}$ for $\Delta$ and $\Lambda$, respectively. These values are similar to those determined by comparing with experiments in this material system [23]. As we can see clearly in Fig. 9, a quite good agreement is achieved. There is only a slight difference near the low temperature end.

\section{CONCLUSIONS}

We have systematically studied intersubband transitions in InAs/AlSb quantum wells with varying temperatures and well widths. The peak frequency decreased with increasing well width and temperature. The decrease with temperature was much larger than what has been observed in GaAs quantum wells. To explain the observations, we developed a comprehensive theoretical model that includes all major many-body effects in combination with an 8-band $\mathbf{k} \cdot \mathbf{p}$ band structure calculation. The theory allows us to study various many-body effects systematically and self-consistently. We observed very interesting interplay of these individual many-body effects. Finally, our theory satisfactorily explains the red shift of the peak position and the increase of linewidth with increasing temperature. A detailed theory-experiment comparison on the temperature dependence of electron density will allow us to develop a more complete understanding of InAs/AlSb quantum wells and a more complete theory with predictive power. Such a theory and the related understanding will allow us to fully explore this materials system in many areas of device applications.

\section{ACKNOWLEDGEMENTS}

This work is supported by DARPA/AFOSR, and by NASA/NCI.

\section{REFERENCES}

[1] C. Mailhiot and D. L. Smith, J. Vac. Sci. Technol. A 7, 445 (1989).

[2] H. Mohseni, E. Michel, Jan Sandoen, M. Razeghi, W. Mitchel, and G. Brown, Appl. Phys. Lett., 71, 1403 (1997)

[3] Many laser related applications based on Antimonide materials can be found in, Antimonide-related strained-layer heterostructures, M.O. Manasreh (ed.), OPA (1997, Amsterdam).

[4] A. Liu and C. Z. Ning, Appl. Phys. Lett. 76, 1984 (2000)

[5] A. Liu and C. Z. Ning, in "Nonlinear Optics: Materials, Fundamentals and Applications", (Optical Society of America, Washington DC, 2000), pp. 56-58. 
[6] G. Tuttle, H. Kroemer, and J. H. English, J. Appl. Phys. 65, 5239 (1989)

[7] H. Kroemer, C. Nguyen, and B. Brar, J. Vac. Sci. Technol. B10, 1769 (1992)

[8] D. J. Chadi, Phys. Rev. B 47, 13478 (1993)

[9] P. J. Lin-Chuang and M. J. Yang, Phys. Rev. B 48, 5338 (1993).

[10] R. J. Warburton, C. Gauer, A. Wixforth, J. P. Kotthaus, B. Brar, and H. Kroemer, Phys. Rev. B 53, 7903 (1996).

[11] T. Ando, Solid State Commun. 21, 133 (1977).

[12] S. J. Allen, D. C. Tsui, and B. Vinter, Solid State Commun. 20, 425 (1976).

[13] S. L. Chuang, M. S.-C. Luo, S. Schmitt-Rink, and A. Pinczuk, Phys. Rev. B 46, 1897 (1992).

[14] D. Huang, G. Gumbs, and M. O. Manasreh, Phys. Rev. B 52, 14126 (1995).

[15] H. Haug and S. W. Koch, Quantum theory of the optical and electronic properties of semiconductors, $2^{\text {nd }}$, World Scientific, (1993, Singapore).

[16] W. Chow and S. W. Koch, Semiconductor-Laser Physics, Spinger-Verlag, (1994, Berlin Heidelberg).

[17] J. Li and C. Z. Ning, to be published.

[18] See, e.g., R. A. Stradling and R. A. Wood, J. Phys. C 3, L94 (1970).

[19] B. C. Covington, C. C. Lee, B. H. Hu, H. F. Taylor, and D. C. Streit, Appl. Phys. Lett. 54, 2145 (1989).

[20] M. O. Manasreh, F. Szmulowicz, D. W. Fischer, K. R. Evans, and C. E. Stutz, App1. Phys. Lett. 57, 1790 (1990).

[21] X. L. Huang et al., J. Appl. Phys. 82, 4394 (1997).

[22] T. Ando, Z. Phys. B 24, 33 (1976).

[23] C. R. Bolognesi, H. Kroemer, and J. H. English, Appl. Phys. Lett. 61, 213 (1992). 